\title{
A low-frequency view of mixed-morphology supernova remnant VRO 42.05.01, and its neighbourhood
}

\author{
M. Arias ${ }^{1}$, J. Vink ${ }^{1,2,3}$, M. Iacobelli ${ }^{4}$, V. Domček ${ }^{1,2}$, M. Haverkorn ${ }^{7}$, J. B. R. Oonk ${ }^{4,5,8}$, I. Polderman ${ }^{7}$, W. Reich ${ }^{6}$,
} G. J. White ${ }^{9,10}$, and P. Zhou ${ }^{1}$

\author{
1 Anton Pannekoek Institute for Astronomy, University of Amsterdam, Science Park 904, 1098 XH Amsterdam, The Netherlands \\ e-mail: maria.arias.de.saavedra@gmail.com \\ 2 GRAPPA, University of Amsterdam, Science Park 904, 1098 XH Amsterdam, The Netherlands \\ 3 SRON, Netherlands Institute for Space Research, Utrech, The Netherlands \\ ASTRON, Netherlands Institute for Radio Astronomy, Postbus 2, 7990 AA Dwingeloo, The Netherlands \\ 5 Leiden Observatory, Leiden University, PO Box 95132300 RA, Leiden, The Netherlands \\ ${ }^{6}$ Max Planck Institute for Radio Astronomy, Auf dem Hügel 69, 53121 Bonn, Germany \\ 7 Department of Astrophysics/IMAPP, Radboud University Nijmegen, PO Box 90106500 GL, Nijmegen, The Netherlands \\ 8 SURFsara, PO Box 946131090 GP, Amsterdam, The Netherlands \\ 9 Department of Physics and Astronomy, The Open University, Walton Hall, Milton Keynes MK7 6AA, UK \\ 10 RAL Space, STFC Rutherford Appleton Laboratory, Chilton, Didcot, Oxfordshire OX11 0QX, UK
}

Received 16 July 2018 / Accepted 7 October 2018

\begin{abstract}
Context. Mixed-morphology supernova remnants (MM SNRs) are a mysterious class of objects that display thermal X-ray emission within their radio shell. They are an older class of SNRs, and as such are profoundly affected by the environment into which they evolve. VRO 42.05.01 is a MM SNR of puzzling morphology in the direction of the Galactic anticentre.

Aims. Low-frequency radio observations of supernova remnants are sensitive to synchrotron electrons accelerated in the shock front. We aim to compare the low-frequency emission to higher frequency observations to understand the environmental and shock acceleration conditions that have given rise to the observed properties of this source.

Methods. We present a LOFAR High Band Antenna map centred at $143 \mathrm{MHz}$ of the region of the Galactic plane centred at $l=166^{\circ}, b=3.5^{\circ}$ at $143 \mathrm{MHz}$, with a resolution of $148^{\prime \prime}$ and an rms noise of $4.4 \mathrm{mJy} \mathrm{bm}^{-1}$. Our map is sensitive to scales as large as $6^{\circ}$. We compared the LOw Frequency ARay (LOFAR) observations to archival higher frequency radio, infrared, and optical data to study the emission properties of the source in different spectral regimes. We did this both for the SNR and for OA 184, an $\mathrm{H}$ II region within our field of view.

Results. We find that the radio spectral index of VRO 42.05.01 increases at low radio frequencies; i.e. the LOFAR flux is higher than expected from the measured spectral index value at higher radio frequencies. This observed curvature in the low-frequency end of the radio spectrum occurs primarily in the brightest regions of the source, while the fainter regions present a roughly constant power-law behaviour between $143 \mathrm{MHz}$ and $2695 \mathrm{MHz}$. We favour an explanation for this steepening whereby radiative shocks have high compression ratios and electrons of different energies probe different length scales across the shocks, therefore sampling regions of different compression ratios.
\end{abstract}

Key words. ISM: supernova remnants - HII regions - ISM: individual objects: VRO 42.05.01 - ISM: individual objects: OA 184

\section{Introduction}

The evolution of supernova remnants (SNRs) is greatly affected by their various environments, which become the dominant factor in the later stages of the life of a remnant. Galactic SNRs are often radio-bright and have large angular scales, allowing us to conduct detailed morphological studies of the sources and their neighbourhood. In particular, the line of sight of the Galactic anticentre towards the Perseus arm is less absorbed and less crowded than a typical Galactic field, while still rich in extended spiral-arm features. Supernova remnants in this direction are excellent sites to study the interaction of a SNR with its surrounding circumstellar and interstellar media. More specifically, the synchrotron emission from their shock-accelerated, relativistic electrons directly probes the acceleration processes that are taking place.
Mixed-morphology supernova remnants (MM SNRs; Rho \& Petre 1998) are a class of SNRs that display a shell-like morphology in the radio but are centrally dominated in the $\mathrm{X}$-rays. Their X-ray emission is characterised by a thermal spectrum and is not due to the presence of a pulsar wind nebula. These objects are in a late stage of SNR evolution in which radiative energy losses become dynamically important. This happens when the post-shock temperature falls below $\sim 5 \times 10^{5} \mathrm{~K}$, which corresponds to shock velocities $V_{\mathrm{s}} \sim 200 \mathrm{~km} \mathrm{~s}^{-1}$, resulting in optical line emission that further cools the remnant (e.g. Blondin et al. 1998). Mixed-morphology SNRs are typically older than 10000 years, although there are a few young MM SNRs: 3C391 is 3700-4400 years old (Chen \& Slane 2001), and W49B has been calculated to be, in one study, 1000-4000 years old (Hwang et al. 2000) and, 


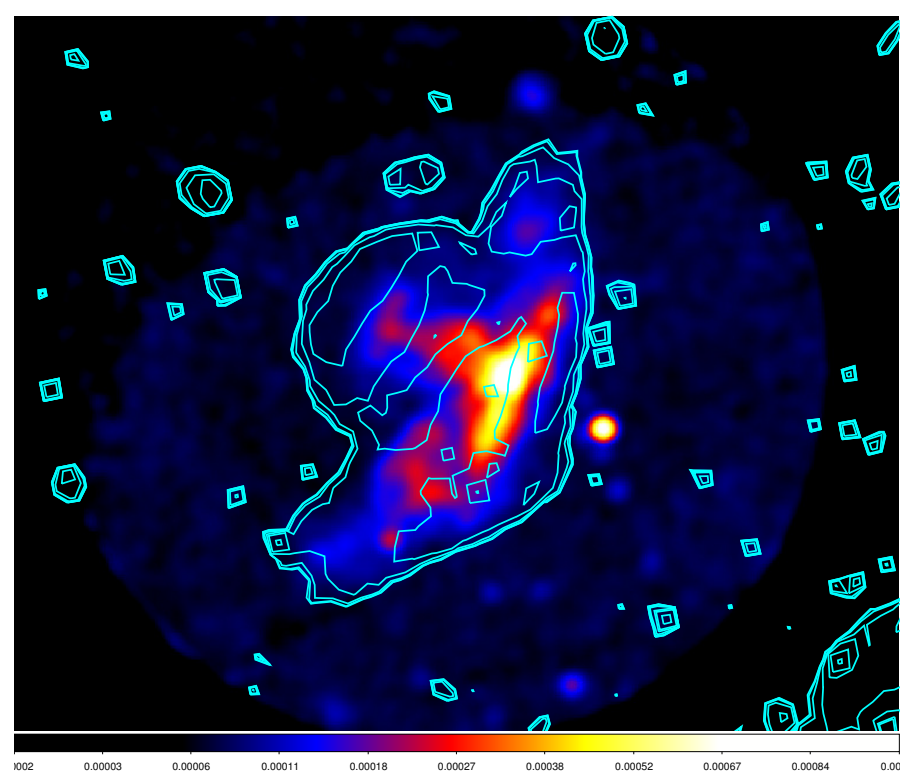

Fig. 1. Exposure-corrected and smoothed X-ray mosaic of VRO 42.05.01 in the $0.1-2.4 \mathrm{keV}$ energy band. The mosaic was constructed from archival ROSAT PSPC observations (RP500001N00 and RP500120N00). The cyan contours correspond to CGPS radio observations at $1420 \mathrm{MHz}$. The $\mathrm{X}$-ray resolution is $1.25^{\prime}$ and the radio resolution is $1.7^{\prime}$.

in another study, 5000-6000 years old (Zhou \& Vink 2018). Moreover, the ages of some of some other MM SNRs have not been clearly determined: IC443 has been proposed to be from 3000-4000 years old to 20000-30000 years old (see e.g. Troja et al. 2008; Gaensler et al. 2006), for example. Another characteristic of MM SNRs is that they tend to have a spectral index flatter than the $\alpha=0.5$ predicted by standard shock acceleration theory (for $S_{v} \propto v^{-\alpha}$; cf. table 4 in Vink 2012). Many of them have been identified to be the result of a core-collapse explosion (Vink 2012), although some might have been thought to be core-collapse remnants because of their mixed-morphology status, and in a few cases a thorough study of the X-ray abundances has indicated that the MM SNR was in fact the result of a type Ia explosion (Yamaguchi et al. 2012; Sezer \& Gök 2012; Zhou \& Vink 2018 for remnants G344.7-0.1, G272.2-3.2, and W49B, respectively).

Remnants of the mixed-morphology class tend to be associated with the denser parts of the interstellar medium (ISM) and often show signs of molecular interaction, as evidenced for instance from $\mathrm{OH}$ maser emission (e.g. IC443, W28, 3C391, etc.; see Table 4 in Vink 2012). This is perhaps to be expected if they are the remnants of the explosions of young massive stars in crowded environments, possibly close to the molecular cloud(s) where the stars were born. In fact MM SNRs are often bright $\mathrm{GeV} \gamma$-ray sources and are the only SNRs for which proton cosmic rays have been unambiguously detected (Ackermann 2013; Giuliani 2011 for the SNRs W44 and IC 443). In X-rays the interiors of MM SNRs show evidence for enhanced abundances (Lazendic \& Slane 2006), indicating the presence of supernova ejecta in addition to the shocked ISM material, and their X-ray emitting plasma tends to be overionised, indicating relatively high densities and rapid adiabatic expansion. These properties suggest that MM SNRs are important for understanding how shock interaction and cosmic-ray acceleration occur in dense environments.

VRO 42.05.01 (G 166.0+4.3) is a MM SNR in the direction of the Galactic anticentre $(\mathrm{RA}=05: 26: 30, \mathrm{Dec}=+42: 56)$. It has a peculiar two-shell radio shape whose outline is filled in X-ray emission (see Figs. 1 and 2). This object displays filamentary optical line emission that is broadly coincident with the outline of the radio shell, and line ratios that suggest a low-density environment (van den Bergh et al. 1973; Fesen et al. 1985). Araya (2013) detected $\mathrm{GeV} \gamma$-ray emission from the source, which, they argued, has a leptonic origin (unlike other MM SNRs). Two targeted pulsar searches were conducted in the region (Biggs \& Lyne 1996; Lorimer et al. 1998), but no pulsar was found. Its distance has been estimated to be $4.5 \pm 1.5 \mathrm{kpc}$, the distance to the Perseus arm at the Galactic latitude of $l=166^{\circ}$ (Landecker et al. 1989).

Our lack of understanding of the morphology of VRO 42.05.01 is twofold: we lack a widely accepted model for the presence of dense X-ray emitting material in the centre of MM SNRs more generally; and it is not clear what conditions in the ejecta and surrounding medium could give rise to the peculiar shape of the source.

The latter has been the subject of speculation for several decades. A series of papers (Landecker et al. 1982; Pineault et al. 1985, 1987; Landecker et al. 1989) proposed that the supernova explosion had taken place in a dense medium, and that a part of the expanding bubble eventually broke into a more tenuous medium, which allowed it to expand at a faster velocity. These papers refer to the smaller semicircle as "the shell", and the triangular larger component as "the wing", a terminology that is maintained throughout the literature (these features are labelled in Fig. 8). Although abrupt density discontinuities in the ISM are common and this scenario is plausible, no unambiguous signs of interaction with the medium have been observed. The relatively low surface density of VRO 42.05.01 ( $7 \mathrm{Jy}$ at $1 \mathrm{GHz}$ for an angular size of $45^{\prime} \times 80^{\prime}$ ) does not seem to agree with the idea that it is evolving in a high-density region. Moreover, the $\mathrm{CO}$ survey of Huang \& Thaddeus (1986) did not show any conspicuous molecular cloud in the face of the remnant, nor clear indications of sharply different densities. On the other hand, the remnant has a flat radio spectral index, $\alpha \approx 0.37$ (Leahy \& Tian 2005). The subject of flat spectral indices in evolved remnants is discussed later in this paper in more detail, but flat radio spectral indices might arise in high compression ratio shocks, suggesting a high post-shock density.

In the case of older, irregular, impossibly-shaped remnants the idea that they might be two SNRs that occurred within a short time is often suggested. The X-ray morphology of VRO 42.05.01, which is internal both to the shell and the wing, would require that both SNRs are MM even though only $\sim 10 \%$ of the Galactic SNR population is MM (Vink 2012). The triangular shape of the larger shell, which has an abrupt ending in a flat line on the east, also disfavours this scenario. However, the possibility that VRO 42.05.01 might be two SNRs has not been completely ruled out.

VRO 42.05.01 has been observed at X-ray wavelengths with ROSAT (Burrows \& Guo 1994), ASCA (Guo \& Burrows 1997), XMM-Newton (Bocchino et al. 2009), and Suzaku (Matsumura et al. 2017). Both Burrows \& Guo (1994) and Guo \& Burrows (1997) found a temperature of $\sim 8.3 \times 10^{6} \mathrm{~K}$ in the X-ray emitting material and remarked on the unusually low column density towards the remnant $\left(2.9 \times 10^{21} \mathrm{~cm}^{-2}\right)$. Bocchino et al. (2009) found that the abundances are well described by a thermal equilibrium model, but the Suzaku observations in Matsumura et al. (2017) are consistent with the presence of a recombining plasma in the smaller shell. The authors proposed that this might be due to heat being conducted from the SNR to a molecular cloud that allegedly sits at the north of the remnant, which lowers the temperature of the SNR. The plasma, they argued, is overionised, as it still has not reached the lower ionisation states associated with cooler temperatures. 


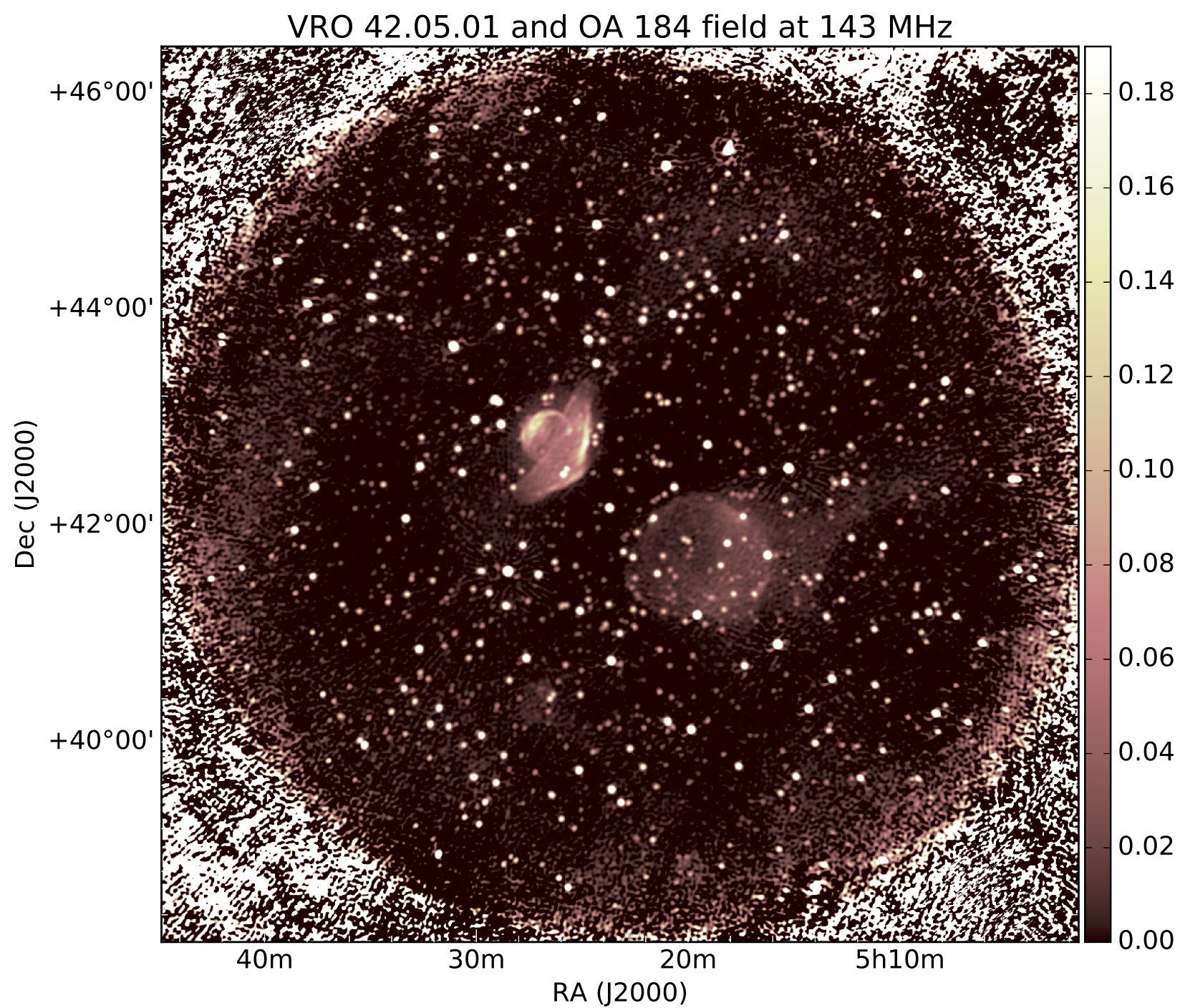

Fig. 2. Full LOFAR field centred at VRO 42.05 .01 and OA 184. This image has a resolution of $148^{\prime \prime}$ and is made using only the core stations for best sensitivity to diffuse, extended emission. It has been primary-beam corrected. The central frequency is $143 \mathrm{MHz}$ and the bandwidth is $59 \mathrm{MHz}$. The noise at the centre of the image is $4.4 \mathrm{mJy} \mathrm{bm}^{-1}$; the colour scale to the right of the map is in Jy bm${ }^{-1}$. VRO 42.05 .01 is the two-shell-shaped extended structure on the left-hand side of the image; the smaller semicircle is the "shell" and the wider semicircle the "wing". OA 184 is the nearly circular shell to the right. The trail of diffuse emission to the west of OA 184 is referred to in the text as the synchrotron tongue.

Table 1. Table of observations.

\begin{tabular}{lc}
\hline \hline Number of stations & 22 \\
Central frequency & $143 \mathrm{MHz}$ \\
Bandwidth & $59 \mathrm{MHz}$ \\
Resolution & $148^{\prime \prime}$ \\
Min baseline & $10 \mathrm{~m}$ \\
Max baseline & $4 \mathrm{~km}$ \\
\hline
\end{tabular}

\section{Data reduction}

\subsection{Observations, calibration, and imaging}

We took eight hours of LOFAR High Band Antenna (HBA) data in January 2016 under project LC5_012. Fifteen minutes at the beginning and the end of the observation were spent on calibrator 3C147, and the remaining time was spent on the field of VRO 42.05.01. For these observations the HBA Dual station configuration was adopted, which is not optimal for calibration and imaging. For this reason, only the visibilities from the core stations were selected and used in the processing. The data were flagged for radio frequency interference, and the data from stations 1 and 13 were removed for the calibrator and the target because they were very noisy.

The 5 Jy source $3 \mathrm{C} 134$ was in the edge of our field of view, approximately 5 degrees from the phase centre. A bright source like this one causes strong artefacts in a large portion of the image, and so we peeled the source (i.e. removed its contribution from the visibilities; Noordam et al. 2004) with the SAGECAL algorithm (Yatawatta et al. 2008).

The calibrator was calibrated in a direction-independent manner using the Pre-Facet Calibration Pipeline (van Weeren et al. 2016). The pipeline obtains diagonal solutions towards the calibrator, 3C147, and then performs clock-TEC separation, which distinguishes between clock offsets and drifts, and signal delays due to the electron column density in the ionosphere. Finally, this pipeline transfers the clock corrections and calibrator gain amplitudes to the target data set.

The visibilities were imaged using the wsclean algorithm (Offringa et al. 2014). The full bandwidth of the LOFAR HBA 


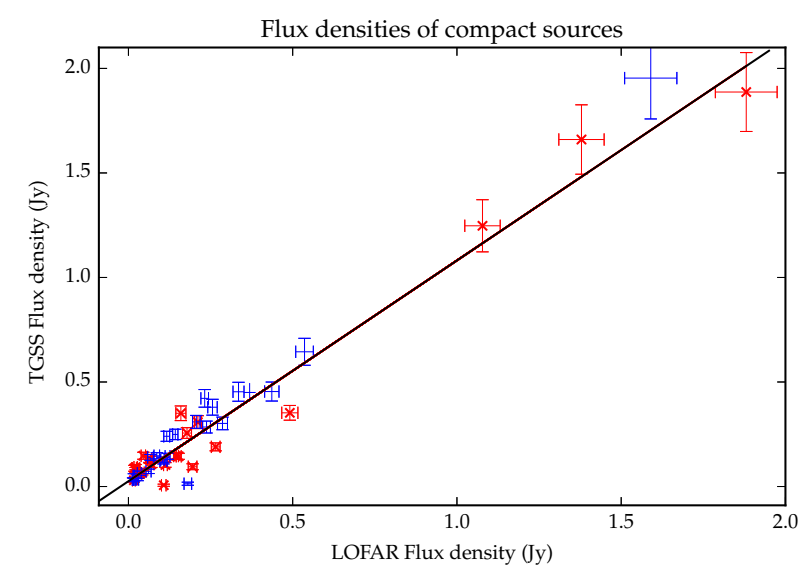

Fig. 3. Comparison of flux density values from the LOFAR HBA images at $143 \mathrm{MHz}$ and the TGSS image at $147 \mathrm{MHz}$ of unresolved sources nearby VRO 42.05.01 (red) and OA 184 (blue). The best-fit line in black has slope $1.05 \pm 0.08$ and intercept $0.025 \pm 0.007$.

was used for making Fig. 2, which was imaged in the multiscale setting and with a Briggs weighting robust parameter of -2 (Briggs 1995). The uniform weighting scheme is possible in the context of these observations because for the LOFAR core stations the baseline distribution is smooth. We imaged using only the LOFAR core stations, resulting in a beam size of $148^{\prime \prime}$. This allowed us to recover the degree-scaled extended emission present in the field. As can be seen in the figure, some bright regions in the extended sources have negative bowls around them owing to the lack of zero spacings.

We pointed the telescope at $\mathrm{RA}=05: 23: 15, \quad \mathrm{Dec}=$ +42.25 .45 , centred between the two large extended sources in the field: the double-shell-shaped VRO 42.05.01 and the H II region OA 184, which is a large ellipse of semimajor axis $80^{\prime}$ and semiminor axis $75^{\prime}$. In addition, in the map we can see some large-scale synchrotron emission parallel to the Galactic plane (see Fig. 12 to see the orientation of the sources in Galactic coordinates). The tail of emission at the west of OA 184, which extends $80^{\prime}$ similar to a comet-tail, was discussed in Leahy \& Tian (2005) and referred to as a "synchrotron tongue" from observations at 408 and $1420 \mathrm{MHz}$. There are two similar tails to either side of the wings of VRO 42.05.01, which are in fact also visible at those higher frequencies (see e.g. the full resolution $1420 \mathrm{MHz}$ map in Fig. 1 of Leahy \& Tian 2005).

\subsection{Total flux density calibration}

As can be seen from Fig. 2, the noise of the image is nonuniform, in part because of the LOFAR beam and in part because of the unmodelled large-scale emission from the Galactic plane. For this reason, we picked $\sim 50$ unresolved sources in the vicinity of VRO 42.05.01 and OA 184 and compared their flux densities in the full bandwidth map at $143 \mathrm{MHz}$ to their flux density in the TIFR GMRT Sky Survey catalogue at $147 \mathrm{MHz}$ (TGSS; Intema et al. 2017). The flux densities as obtained from the HBA and TGSS images are plotted in Fig. 3.

As one can see from this plot, LOFAR measures flux density values for these compact sources that are systematically lower than those of TGSS by a factor of 0.95 . Differences in flux densities measured by LOFAR and the GMRT have been noted in van Weeren et al. (2016) and Shimwell et al. (2017). Our small difference of a factor of 0.95 is well within the uncertainty in the calibration of the low-frequency flux scale with the GMRT
Table 2. Flux densities, LOFAR in-band spectral index, and LOFARCGPS spectral index, with and without compact source (CS) subtraction.

\begin{tabular}{lcc}
\hline \hline Source & VRO 42.05.01 & OA 184 \\
\hline$S_{143 \mathrm{MHz}}$ with CS & $16.4 \pm 0.1 \mathrm{Jy}$ & $18.3 \pm 0.1 \mathrm{Jy}$ \\
$S_{143 \mathrm{MHz}}$ CS subtracted & $15.8 \pm 0.1 \mathrm{Jy}$ & $11.2 \pm 0.2 \mathrm{Jy}$ \\
In-band $\alpha$ with CS & $0.58 \pm 0.04$ & $0.48 \pm 0.02$ \\
In-band $\alpha$ CS subtracted & $0.57 \pm 0.04$ & $0.37 \pm 0.02$ \\
$\alpha$ LOFAR-CGPS with CS & $0.49 \pm 0.02$ & $0.32 \pm 0.01$ \\
$\alpha$ CS subtracted & $0.49 \pm 0.02$ & $0.16 \pm 0.01$ \\
$\alpha$ L\&T05 with CS & $0.38 \pm 0.03$ & $0.28 \pm 0.10$ \\
$\alpha$ L\&T05 CS subtracted & $0.36 \pm 0.03$ & $0.21 \pm 0.12$ \\
\hline
\end{tabular}

Notes. For comparison we also include the spectral indices from Leahy \& Tian (2005).

(estimated to be around 10\%, Intema et al. 2017). Moreover, the systematic calibration errors in the LOFAR flux scale are of the order of $10 \%$, which can explain the difference. We note that we are comparing two nearby but not identical frequencies (143 MHz for LOFAR and $147 \mathrm{MHz}$ for the GMRT). Rescaling the LOFAR flux densities to the TGSS frequency with a spectral index of 0.8 , typical for extragalactic sources, makes the LOFAR flux densities systematically lower than the TGSS flux densities by a factor of 0.93 instead of 0.95 , which is still within the uncertainty in the low-frequency flux scale.

In Table 2 we present the flux density values for VRO 42.05.01 and OA 184 as measured with LOFAR, as well as the in-band spectral index for each source (see the next section). Both of the extended sources are covered in point sources. We present the flux density measurements for each source both subtracting the point sources that are visible in the face of the extended source and without this subtraction, and we do the same thing for the spectral index. In the case of VRO 42.05.01 we subtract the two bright sources evident in the wing. The third remaining point source over the remnant discussed in Leahy \& Tian (2005) is confused at LOFAR frequencies within the extended emission of the remnant. In the case of OA184 we subtract the 26 sources above $14 \mathrm{mJy} \mathrm{bm}^{-1}$ ( $\sim 3 \sigma$ of the image noise) that are visible in the face of the source.

\subsection{Narrow-band images and in-band spectral index}

Since our HBA observations covered a large bandwidth (from $115 \mathrm{MHz}$ to $174 \mathrm{MHz}$ ), we can obtain spectral information from our pointing. Shimwell et al. (2018) have discussed that it is possible to divide the LOFAR HBA bandwidth into three parts, make a three channel image, and accurately derive (with $10 \%$ uncertainty or less) in-band spectra for sources with integrated flux densities $\geq 5 \mathrm{mJy}$ (for maps with a typical rms of $0.1 \mathrm{mJy} \mathrm{bm}^{-1}$ ).

For that reason, we divided the bandwidth of our data to make three $20 \mathrm{MHz}$ wide images centred at 125,144 , and $164 \mathrm{MHz}$ (see Fig. A.1). These were made with a common uvrange of $10 \lambda-1400 \lambda$.

We report the value of the integrated spectral indices of the extended sources, both with and without compact source subtraction, in Table 2. To calculate these we fit a power law to the flux density values at the three frequencies. We note that the inband spectral index is calculated from three frequencies at 125 , 144 , and $164 \mathrm{MHz}$, and so it does not have much of a leverage arm in frequency and is severely subject to LOFAR systematics. We therefore also calculated the spectral index including the flux densities from the Canadian Galactic Plane Survey (CGPS; 

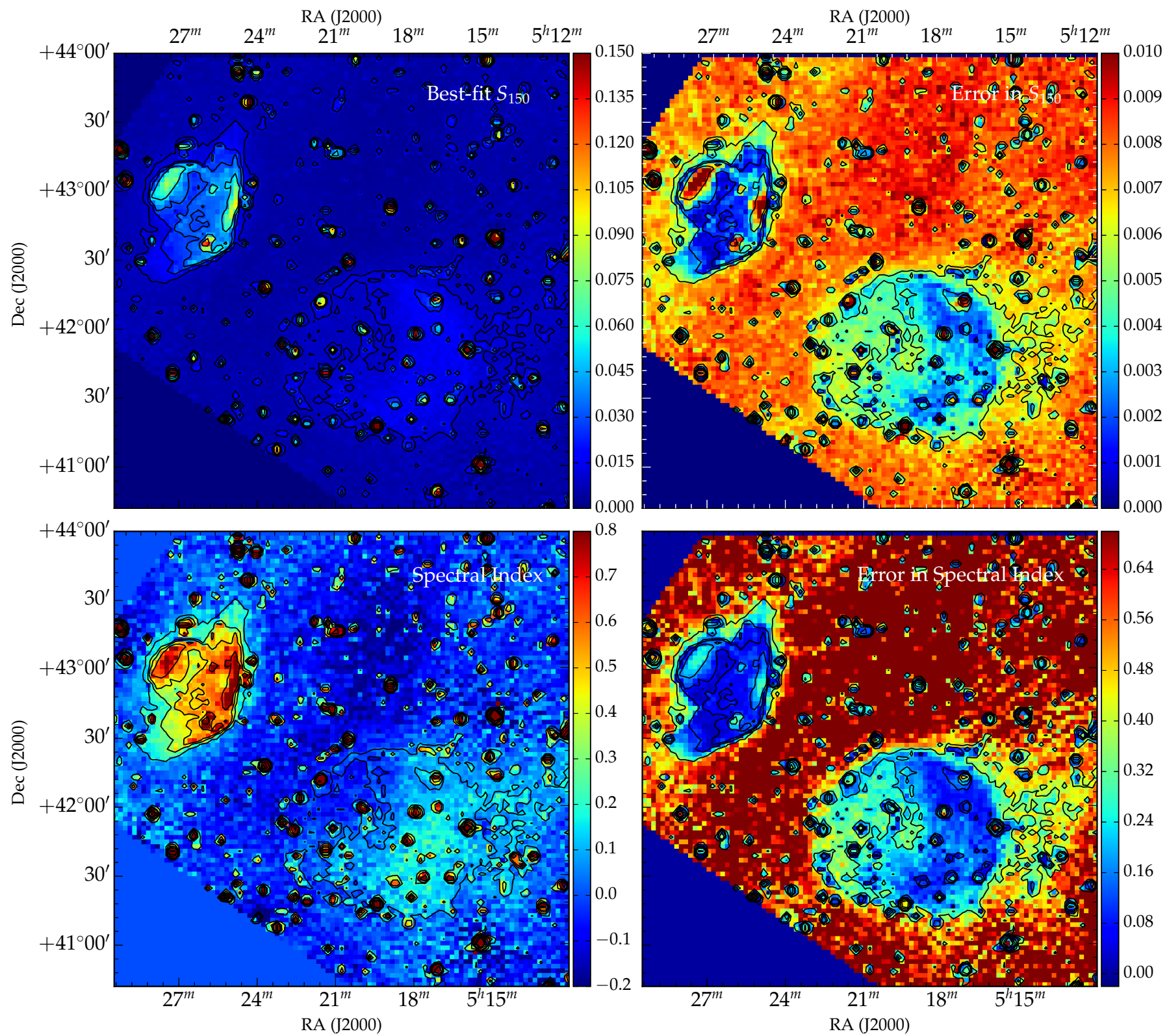

Fig. 4. Results of fitting LOFAR, CGPS, and Effelsberg data to Eq. (1). Top left: best-fit amplitude $S_{150 \mathrm{MHz}}$ and top right: error in best-fit amplitude $\Delta S_{150 \mathrm{MHz}}$. Bottom left: best-fit spectral index value $\alpha$ and bottom right: error in spectral index $\Delta \alpha$. The contours are those of the best-fit amplitude $S_{150 \mathrm{MHz}}$

Taylor et al. 2003) images as reported in Leahy \& Tian (2005). In this case we fitted the flux density values in Table 2 and the $408 \mathrm{MHz}$ and $1420 \mathrm{MHz}$ values in Table 3 of Leahy \& Tian (2005) to a power law.

The spectral index values that we find are different to those in Leahy \& Tian (2005), which were calculated between $408 \mathrm{MHz}$ and $1420 \mathrm{MHz}$. The only value that agrees within the error bars is the spectral index of OA 184 without compact source subtraction. This is in fact surprising, as extragalactic compact sources typically have $\alpha \sim 0.7-0.8$ (Mahony et al. 2016; Williams et al. 2016), and so one would expect their contribution to steepen the overall spectrum at LOFAR frequencies. If we subtract the compact sources, our value of $\alpha=0.16 \pm 0.01$ is in fact smaller than that found in Leahy \& Tian (2005). For an optically thin HII region, $\alpha=0.16$ is a reasonable spectral index value and part of the discrepancy with Leahy \& Tian (2005) might be due to our different threshold values for removing the compact sources: they remove
17 sources with flux densities as low as $20 \mathrm{mJy}$ at $408 \mathrm{MHz}$, whereas we remove 26 sources above $14 \mathrm{mJy}$ at $143 \mathrm{MHz}$.

On the other hand, we find VRO 42.05.01 to have a significantly steeper index than previously reported. The LOFAR inband spectral index ( $\alpha=0.58$ and 0.57 with and without compact source subtraction, respectively) is steeper than the spectral index if we include the higher frequency flux densities $(\alpha=0.49$ and 0.49), and both are much steeper than the spectral index found only from the CGPS data (0.37 and 0.36). We explore several scenarios that could account for an intrinsic steepening of VRO 42.05.01 at LOFAR frequencies in Sect. 5.

We made an in-band spectral index map from these three narrow-band images. It is presented in Fig. 6, upper left. We note that the LOFAR in-band spectral index map is not trustworthy since the side lobes are not calibrated as a function of elevation. The primary beam can also introduce spurious effects that affect the in-band spectral index radially. In addition to these 
instrumental effects, the large fluctuations in the in-band spectral index are also affected by artefacts in the narrow-band images (such as bowls around the bright sources). The oscillations are of course also larger in the areas of poorer signal to noise. For these reasons, the backbone of our analysis is comparing the LOFAR map to higher frequency observations.

\subsection{Other data and higher frequency spectral index map}

In this paper we compare our LOFAR observations to higher frequency archival data. At radio frequencies, we use the $408 \mathrm{MHz}$ and $1420 \mathrm{MHz}$ continuum maps from the CGPS (Taylor et al. 2003) as well as the $2695 \mathrm{MHz}$ maps from the Effelsberg $11 \mathrm{~cm}$ Survey in the direction of the Galactic anticentre (Fürst et al. 1990). The Effelsberg $11 \mathrm{~cm}$ data has a resolution of $4.3^{\prime}$. The CGPS samples baselines from 12.9 to $604.3 \mathrm{~m}$, which correspond to scales of $1.7^{\prime}-56^{\prime}$ at $1420 \mathrm{MHz}$, and $4.1^{\prime}-195^{\prime}$ at $408 \mathrm{MHz}$. This survey also includes single-dish data to account for zero spacings. For comparison, our LOFAR images are sensitive to scales of $\sim 3^{\prime}-360^{\prime}$. VRO 42.05 .01 and OA184 present substantial amount of diffuse emission on degree scales, but within the scales probed by LOFAR. Therefore, the LOFAR, CGPS, and Effelsberg data can be safely compared.

We smoothed the $143 \mathrm{MHz}, 408 \mathrm{MHz}$, and $1420 \mathrm{MHz}$ to the $4.3^{\prime}$ resolution of the $2695 \mathrm{MHz}$ Effelsberg map and made a spectral index map by fitting each pixel in each of the images to a power law of the following form:

$S_{v}=S_{150 \mathrm{MHz}}\left(\frac{v}{150 \mathrm{MHz}}\right)^{-\alpha}$,

where our fit parameters were the flux density at $150 \mathrm{MHz}$ $S_{150 \mathrm{MHz}}$ and the spectral index $\alpha$. The best-fit spectral index, flux densities, and errors in each for VRO 42.05.01 and OA184 are plotted in Fig. 4.

In addition to the continuum data products, we use $21 \mathrm{~cm}$ data cubes from the CGPS to study the neutral hydrogen environment of these sources. At infrared wavelengths we use data from the Wide-Field Infrared Survey Explorer all-sky survey (WISE; Wright et al. 2010). Finally, we took the optical $\mathrm{H} \alpha$ map from the Middlebury Emission-Line Atlas of Galactic SNRs, found online $^{1}$.

\subsection{Curvature in the radio spectrum of VRO 42.05 .01}

The best-fit radio surface brightness at $150 \mathrm{MHz}\left(S_{150}\right.$, Fig. 4 upper left corner) is lower than that in our LOFAR data. This is of course because the integrated surface brightness at $143 \mathrm{MHz}$ is substantially higher than predicted from the higher frequency data. The spectrum has curvature and so cannot be accurately fit by a power law; we discuss this curvature thoroughly in later sections of this paper.

In fact, the spectral index of an $\mathrm{H}$ II region also changes as it goes from being optically thin to optically thick. This could presumably happen at LOFAR frequencies and so, in principle, there is no reason why a power law should describe the spectrum of OA 184 in the frequencies of relevance for this paper.

For this reason we made multiple spectral index maps using different combinations of the LOFAR, CGPS $408 \mathrm{MHz}$, CGPS $1420 \mathrm{MHz}$, and Effelsberg $2695 \mathrm{MHz}$ maps. These are shown in Fig. 6.

\footnotetext{
1 http://sites.middlebury.edu/snratlas/ g166-04-3-vro-42-05-01/ (Winkler et al., in prep.)
}

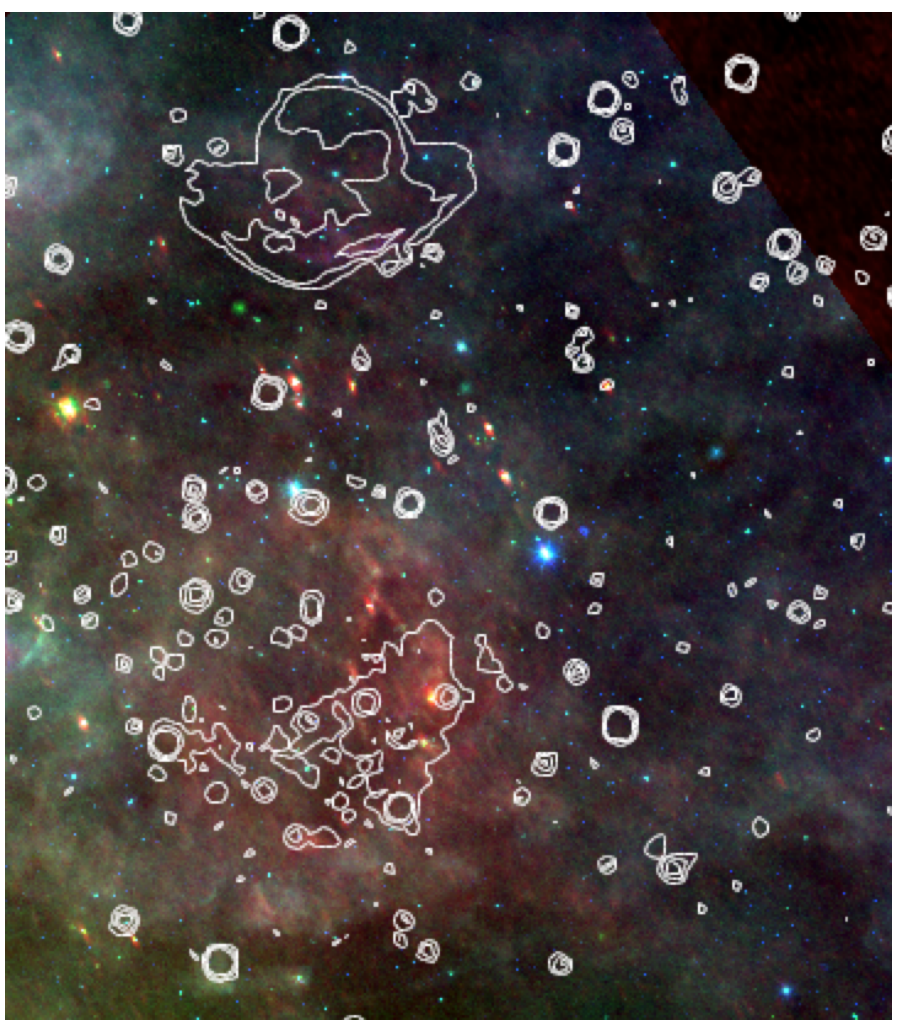

Fig. 5. Three colour image made from IRAS $60 \mu \mathrm{m}$ image (red), Wise $22 \mu \mathrm{m}$ (green) and Wise $12 \mu \mathrm{m}$ (blue). The contours are from the LOFAR full bandwidth image.

\section{OA 184}

Although in earlier works OA 184 has been considered to be a SNR, Foster et al. (2006) presented compelling evidence that it is, in fact, an H II region, and OA 184 was subsequently removed from the Green SNR Catalogue (Green 2017). Foster et al. (2006) cited the following reasons for OA 184 not being a SNR:

- It shows no X-ray emission.

- It is not seen in low-frequency surveys (e.g. Vessey \& Green 1998) because of a flat radio spectrum ( $\alpha=0.2$ to $\alpha=0.14)$.

- It presents in IR surveys as a shell (see e.g. Fig. 5).

- In the optical spectrum $\mathrm{H} \alpha$ dominates over other metal line emission (Fesen et al. 1985).

- There is no polarised continuum emission at $21 \mathrm{~cm}$ (Kothes et al. 2006).

- The radio recombination hydrogen lines are at a level consistent with a thermal shell (Foster et al. 2006).

- There is a single 07.5V star within the shell with a similar excitation parameter as the nebula (Foster et al. 2006).

The lack of X-ray emission could be consistent with a scenario in which OA184 is a SNR that is too old to emit X-rays. However, the lack of forbidden line emission from metals, the bright, clumpy infrared emission, and the recombination lines make it difficult to argue against the case that OA 184 is an H II region, likely energised by its central star.

Our LOFAR observations agree very well with the flat (thermal) radio spectrum derived by Foster et al. (2006). As mentioned by Leahy \& Tian (2005) and Foster et al. (2006) much of the integrated flux density measurement difficulty has to do with subtracting the emission from compact sources in the face of the shell. We subtracted more sources from the total flux density of 
M. Arias et al.: A low-frequency view of mixed-morphology supernova remnant VRO 42.05.01, and its neighbourhood
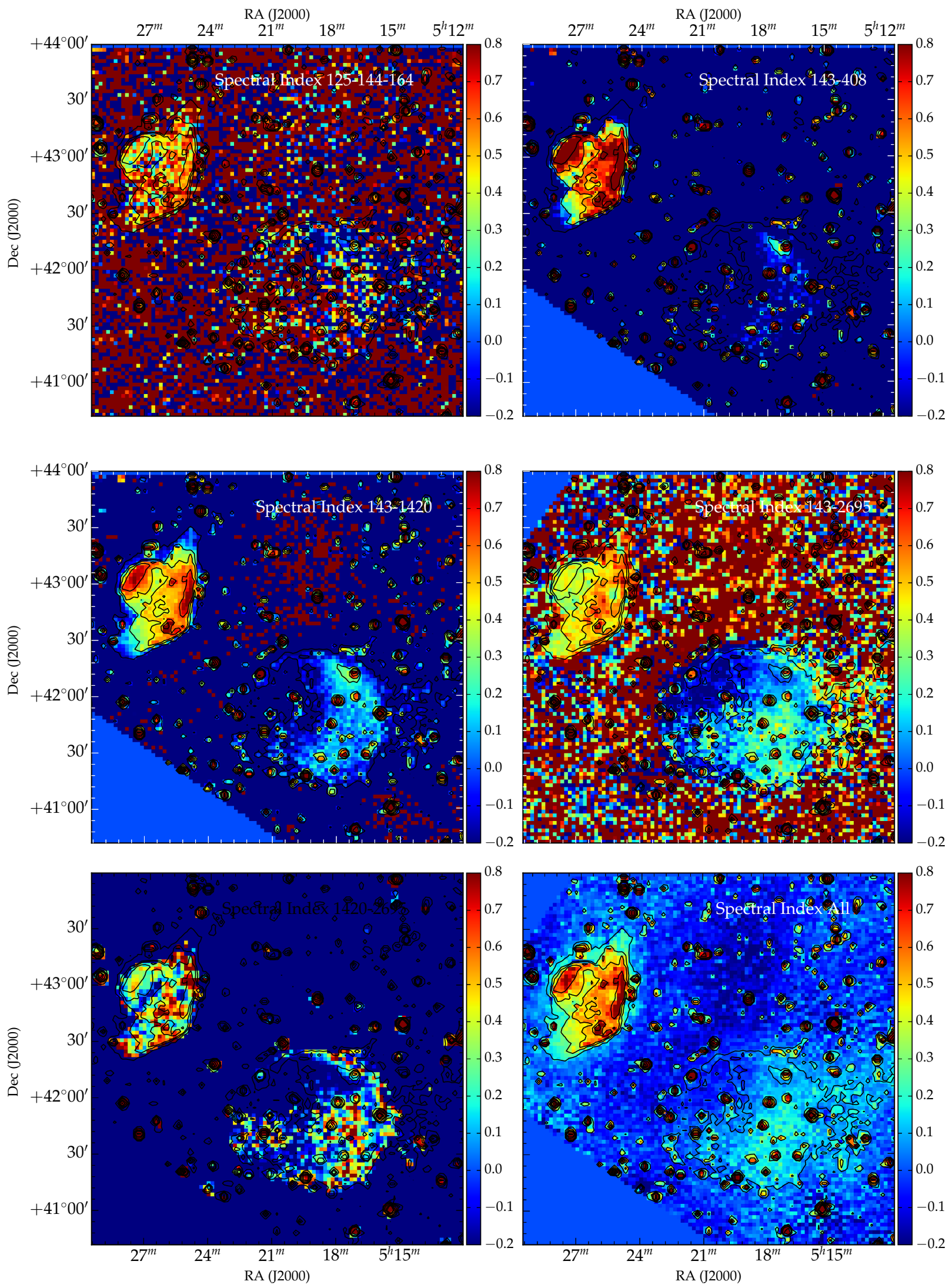

Fig. 6. Spectral index maps made from the LOFAR, CGPS, and Effelsberg data. Top left: LOFAR in-band spectral index made from the three narrow-band images described in Sect. 2.3 and presented in the appendix. Top right: $143 \mathrm{MHz}-408 \mathrm{MHz}$ (LOFAR to CGPS) spectral index. Middle left: $143 \mathrm{MHz}-1420 \mathrm{MHz}$ (LOFAR to CGPS) spectral index. Middle right: $143 \mathrm{MHz}-2695 \mathrm{MHz}$ (LOFAR to Effelsberg) spectral index map. Bottom left: spectral index from CGPS and Effelsberg data $(1420 \mathrm{MHz}-2725 \mathrm{MHz})$. Bottom right: spectral index map combining all frequencies, as presented in Fig. 4. 


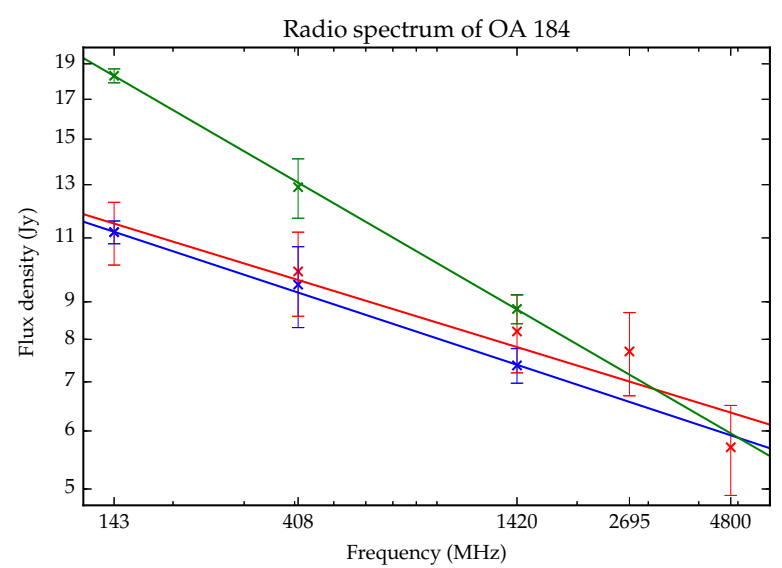

Fig. 7. Flux density values of OA 184 including compact sources (green), with the compact sources subtracted as described in Foster et al. (2006) (red), and with the compact sources subtracted as extrapolated from the total flux density in compact components as measured with LOFAR (blue).

the OA 184 outline than the earlier works because these typical sources have a spectrum $\alpha \sim 0.7$ and therefore are more prominent in the LOFAR image. We accounted for these differences in the following way: in our LOFAR image, we measured 7.1 Jy in compact sources. For a spectral index $\alpha=0.7$ we expect these to contribute $3.4 \mathrm{Jy}$ at $408 \mathrm{MHz}$ and $1.4 \mathrm{Jy}$ at $1420 \mathrm{MHz}$. We subtracted these values to the total (OA 184 + compact sources) flux density values quoted in Leahy \& Tian (2005). The fits for the flux density values of OA 184 with compact sources, with the compact sources subtracted as described in Foster et al. (2006), and with the compact sources subtracted as described above are plotted in Fig. 7.

\subsection{Structures within OA 184}

An aspect worth noting regarding the morphology of this source is that both the radio intensity (Fig. 2) and the spectral index behaviour at all frequencies (Fig. 6) suggest that there are three distinct regions in OA 184: a faint region to the east, a brighter, crescent-moon shape in the west, and a small bright spot in the north. This crescent moon-shaped feature is also responsible for the bulk of infrared emission from OA 184 (see Fig. 5), and has substantial morphological correspondence with the neutral hydrogen emission seen at velocities of $\sim 27-36 \mathrm{~km} \mathrm{~s}^{-1}$.

Figure 8 shows the behaviour with frequency of the crescentmoon shaped region and a bright spot in the north of the source. In the case of the crescent, the H II emission becomes optically thick at around $400 \mathrm{MHz}$, whereas the bright region becomes optically thick at a lower frequency between the $143 \mathrm{MHz}$ and $408 \mathrm{MHz}$ points. This can explain the diversity in spectral index behaviour for these regions in Fig. 6.

\subsection{The synchrotron tongue}

Foster et al. (2006) proposed that the "tongue", the extended trail west of OA184, is a synchrotron filament that lies behind the $\mathrm{H}$ II region shell. They saw polarised $6 \mathrm{~cm}$ emission both inside OA184 and to its west, where the tongue is located, and there is a gap coincident with the edge of the shell. They argued that the shell depolarises the background polarised emission from the tongue. At the rim of the shell, where it is the thickest, is where the majority of the depolarisation occurs. They invoke a shell
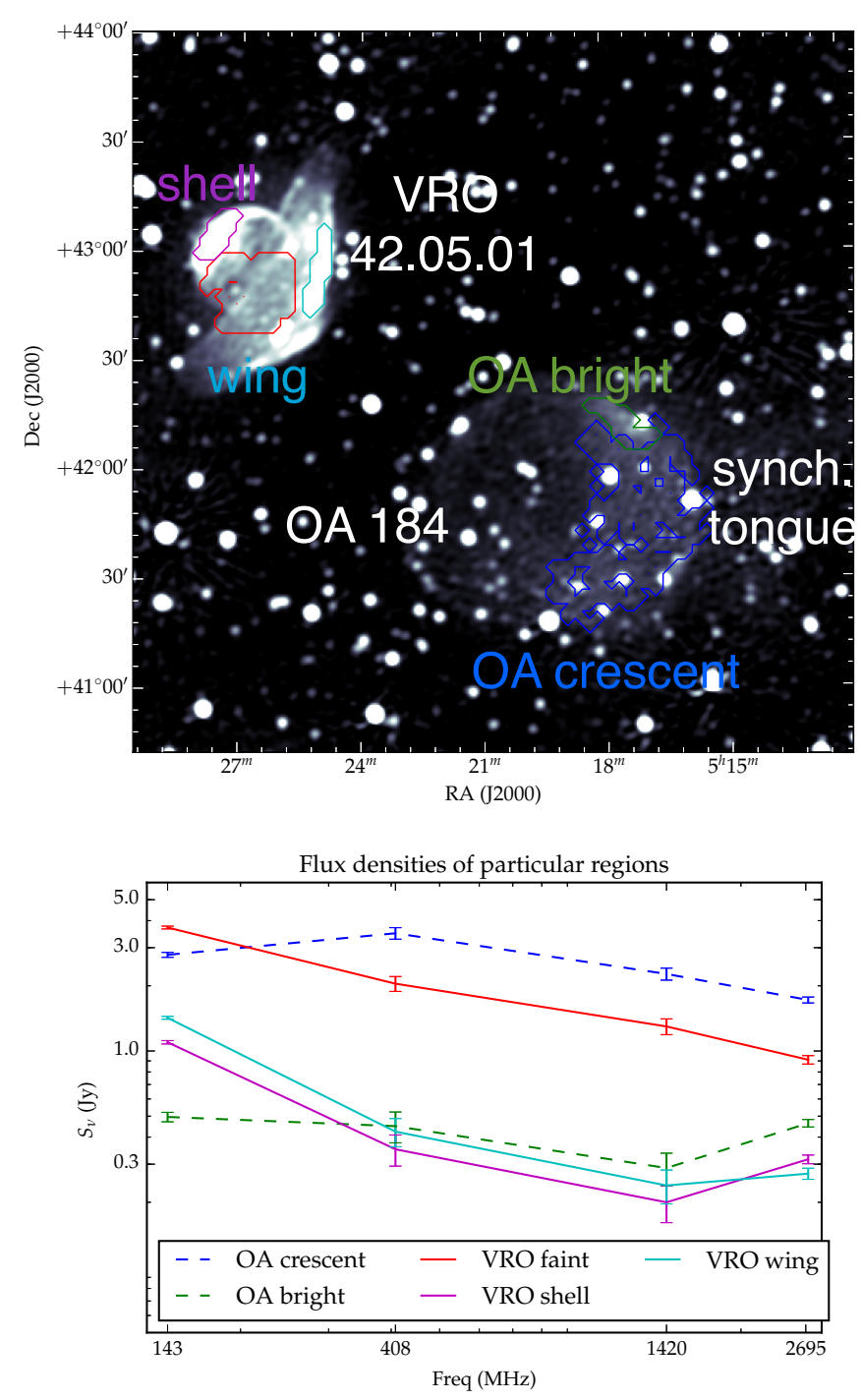

Fig. 8. Flux density values of some regions in OA 184 and VRO 42.05.01. The colours in the plot correspond to the regions of the same colour in the map above.

structure of thickness $\sim 0.11^{\circ}$ to account for the necessary rotation measure.

Our LOFAR observations show no limb brightening, which is expected if OA 184 were indeed such a shell. In fact, from our observations, it seems that the tongue does not emit synchrotron at all.

In most of the spectral index maps in Fig. 6 one cannot see the tongue from the background. However, in the spectral index map including all the data (Fig. 4), the tongue has a spectral index behaviour similar to the brighter, crescent-shaped region of OA 184. In comparing our Fig. 2 to the full resolution $1420 \mathrm{MHz}$ map in Fig. 1 of Leahy \& Tian (2005), we can appreciate that the tongue is not any more prominent at $143 \mathrm{MHz}$ than at $1420 \mathrm{MHz}$. In fact, in addition to its flat spectral index, the tongue west of OA 184 displays a substantial amount of infrared emission (see Fig. 5), suggesting a thermal origin. This component could be linked to the structure that appears in $\mathrm{H} \mathrm{I}$ at -25 to $-33 \mathrm{~km} \mathrm{~s}^{-1}$ (see Fig. 12). For these reasons we suggest that the tongue might be a $\mathrm{H}$ II region.

The similarities in spectral index behaviour and infrared morphologies, as well as the fact that both structures appear and 


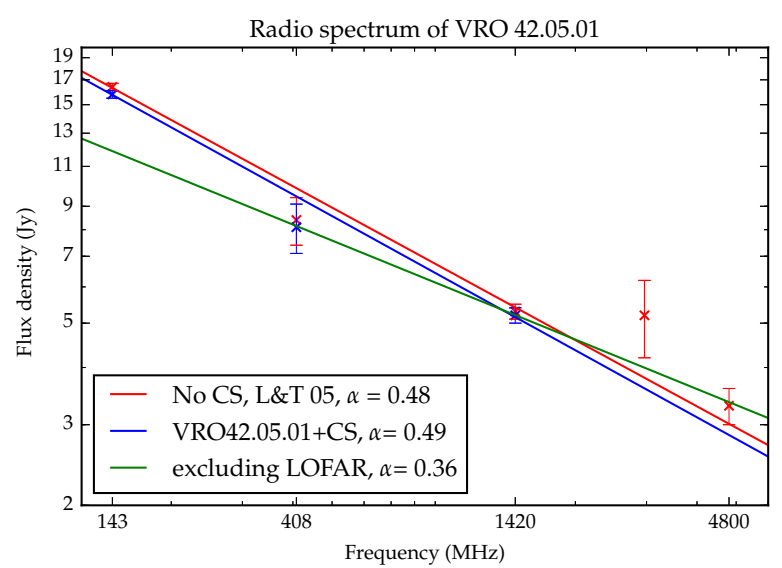

Fig. 9. Flux density values of VRO 42.05 .01 including compact sources (blue), with the compact sources subtracted as described in Leahy \& Tian (2005) (red), and excluding the $143 \mathrm{MHz}$ LOFAR data point (green). The point at $4800 \mathrm{MHz}$ is taken from Gao et al. (2011) and does not have the compact sources subtracted.

fade at similar velocity ranges, could suggest that OA 184 and the tongue are in fact part of the same structure; for instance, the tongue could be a breakout of the $\mathrm{H}$ II region or could have that shape owing to some projection effect. However, Foster et al. (2006) found different polarisation properties in both structures, which complicates the suggestion that they might be a single H II region.

\section{Analysis of VRO 42.05 .01}

\subsection{Comparisons with earlier work}

The LOFAR observations show that VRO 42.05 .01 is significantly brighter at $143 \mathrm{MHz}$ than the earlier spectral index calculations predict (see Fig. 9, plus spectral index calculations in Leahy \& Tian 2005; Gao et al. 2011). Even accounting for the presence of compact sources in the face of the remnant it is clear that the radio spectrum of VRO 42.05.01 steepens at low frequencies. We present possible interpretations for the curved spectrum of the source later in this paper (Sect. 5).

Leahy \& Tian (2005) used the $1420 \mathrm{MHz}$ and 408 CGPS data to conduct a thorough study of the radio spectrum of VRO 42.05.01 and OA 184. These authors found that for VRO 42.05.01 the shell and wing regions have different spectral indices: $\alpha_{\text {shell }}=0.31 \pm 0.03$ and $\alpha_{\text {wing }}=0.47 \pm 0.03$. This difference is noticeable in Fig. 6, bottom left. The LOFAR-Effelsberg map (Fig. 6, bottom right), which has the largest leverage arm in frequency, gives $\alpha_{\text {shell }}=0.41 \pm 0.02$ and $\alpha_{\text {wing }}=0.48 \pm 0.03$; these are slightly larger indices than the Leahy \& Tian (2005) values, but preserve the difference between shell and wing.

We suspect that the difference in spectral index behaviour between the shell and the wing is in fact due to a difference between faint and bright components. The plot in Fig. 8 shows the flux densities at the frequencies of interest of three regions in VRO 42.05.01: the two very bright regions in the north of the shell and the west of the wing, and a diffuse, faint component that extends between both. The two brightest regions in the shell and the wing have very similar behaviour with a flat spectral index at higher frequencies and an abrupt steepening at LOFAR frequencies. The faint component has a roughly constant powerlaw shape with slope $\alpha_{\text {faint }}=0.48 \pm 0.02$.

It is possible that the faint region has the same steepening as the bright regions at LOFAR frequencies but that it is not visible

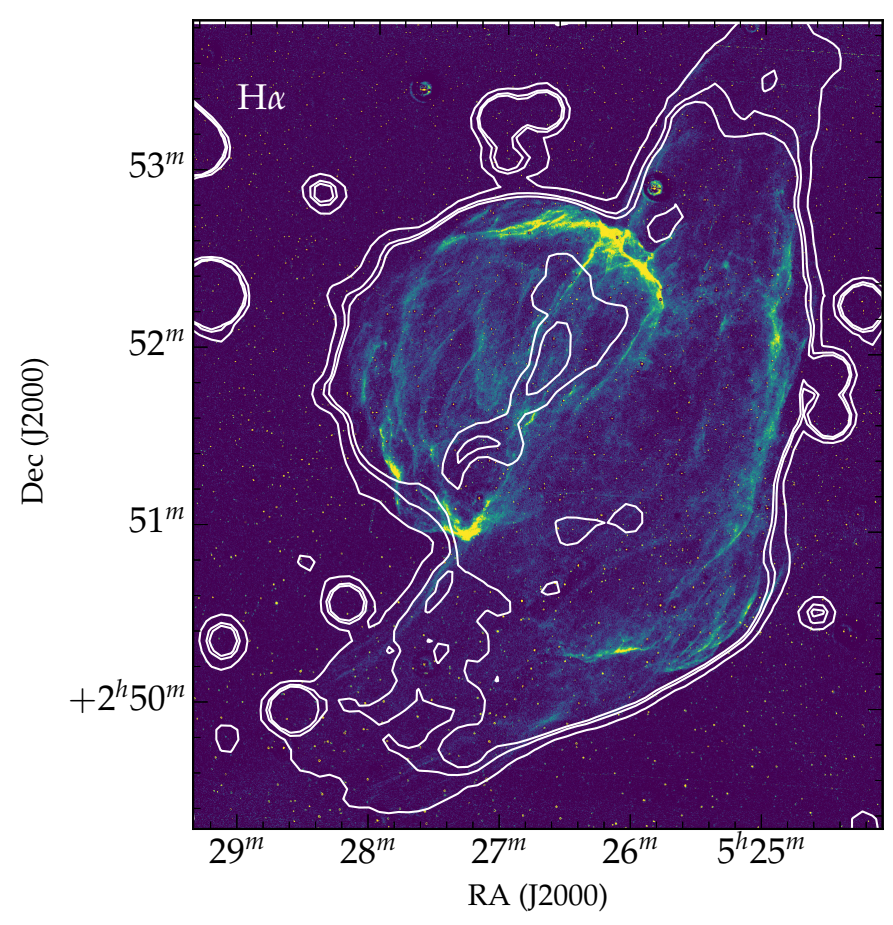

Fig. 10. H $\alpha$ map overlaid with $143 \mathrm{MHz}$ contours. Taken from the Middlebury Emission-Line Atlas of Galactic SNRs (Winkler et al., in prep.).

because of free-free absorption. This would require there being ionised gas covering the surface area of only the red region in Fig. 8, and not the cyan nor the magenta; this is unlikely since the X-ray papers (Burrows \& Guo 1994; Guo \& Burrows 1997) have found a very low column density towards VRO 42.05.01. Moreover, for free-free absorption to be significant at $150 \mathrm{MHz}$, the purported ionised gas would have to have a high emission measure, which would likely result in radio, optical, or infrared emission. We believe that the difference in spectral behaviour of steep and faint components is intrinsic and due to the shock physics at play, which we discuss in Sect. 5.

\subsection{Relation between radio spectral index, radio brightness, and $\mathrm{H} \alpha$ brightness}

In the case of radiative SNRs, optical emission traces lowdensity atomic gas that is rapidly cooling. Fig. 10 shows the morphology of VRO 42.05.01 in $\mathrm{H} \alpha$ emission. It is striking how much the optical morphology resembles the radio morphology, outlining both the wing and the shell. Fig. 11 further emphasises this point, showing a roughly constant ratio of optical to radio emission, save for a region of excess optical emission in the line where the shell and the wing meet.

In general, the coincidence of the synchrotron-enhanced radio emission with very strong optical filaments in lines suggests that the optical features delineate the position of cooling postshock ISM gas. The region of excess optical emission along with the presence of the two very bright optical knots in the junction where the two structures cut suggests that we are looking at a circular rim that is perpendicular to the plane of the sky and has swept a substantial amount of hydrogen but is rather thin (and so emits little synchrotron radiation relative to the rest of the remnant). It is possible to think of a sphere (the shell) and a cone (the wing) that meet in this optically emitting rim that is a boundary between both structures and the ISM. If this is the case, then the optical and radio emission in the wider side of the wing require 


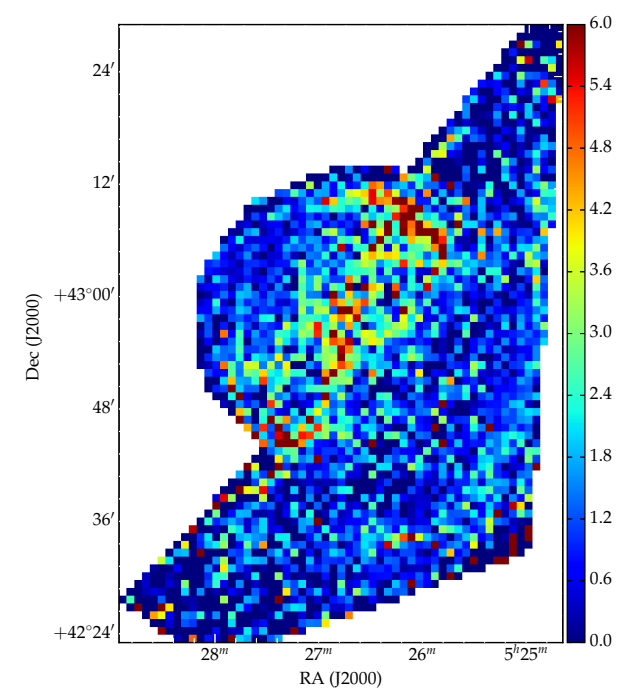

Fig. 11. Ratio of optical to radio emission. The $\mathrm{H} \alpha$ map in Fig. 10 was resolved down to match the radio image, and the emission within the masked region of VRO 42.05.01 was normalised to one for both the optical and radio maps before taking the ratio.

that the base of the cone has a sharp edge, which is difficult to form if the wing is the result of a shock breaking into a region of lower density, as suggested by Pineault et al. (1985).

The region of excess optical emission, moreover, appears to have a flatter spectral index than the rest of the SNR in all the maps in Fig. 6. Although from the optical map the emission in the boundary between the shell and the wing appears to be a limb brightening effect, it is not clear why the limb brightening would not affect the radio emission. There must be some additional physical effect at play that enhances the optical emission or suppresses and/or flattens the synchrotron emission.

\subsection{Environment and interaction}

There are several important hints suggesting that VRO 42.05.01 might be evolving within an environment of different densities. Landecker et al. (1982, 1989), and Pineault et al. (1985, 1987) all invoked an inhomogeneous medium to explain the unusual morphology of VRO 42.05.01. The latter paper analyses interferometer and single-dish data of $21 \mathrm{~cm}$ line emission in the line of sight of VRO 42.05.01 and identifies multiple features at different velocities that seem to match the morphology of the source and that, according to the authors, establish interaction. The Suzaku observations in Matsumura et al. (2017) show that the X-ray spectrum of the shell side of the remnant is described by a recombining plasma, and the authors argue that it is because in this side the remnant is in contact with cool, dense gas. They also point out that the emission measure suggests higher ambient gas density in the shell region. Leahy \& Tian (2005), who found that the shell has a lower spectral index than the wing, suggested that different Mach number values in each of the shock fronts are responsible for this feature. In this case, the lower spectral index for the shell would be explained by a higher density surrounding it.

There are also some reasons to be suspicious that these inhomogeneous density conditions do in fact describe the environment of VRO 42.05.01. One reason is that the surface brightness of VRO 42.05.01 is relatively low for an SNR evolving in a dense medium. Secondly, the $\gamma$-ray spectrum in Araya (2013) is best explained by inverse Compton emission from relativistic electrons, and not from the decay of neutral pions produced from accelerated cosmic rays interacting with a nearby molecular cloud. Also, the Landecker et al. (1989) argument is based on morphological similarities of the H I and SNR features, which, although suggestive, are no physical proof of interaction. The CO survey of Huang \& Thaddeus (1986) did not show signs of sharply differing densities around the remnant, nor any clear molecular cloud. To our knowledge, there has been no study of molecular line spectra in the direction of the SNR for broadenings, wings, asymmetries, or ratios of different excitation states in the lines, which would constitute an unambiguous proof that the SNR is interacting with a nearby molecular cloud.

The CGPS included an atomic hydrogen survey made with the Dominion Radio Astronomical Observatory (DRAO) interferometer complemented with data from the DRAO $26 \mathrm{~m}$ single dish. Incidentally, this is the same telescope set-up used in the Landecker et al. (1989) paper, whose data is sensitive to all structures of angular scales down to their $4^{\prime}$ resolution. By the time the CPGS data was taken, the interferometer had gone through several upgrades, resulting in a higher angular resolution of $1.7^{\prime}$.

Having said that, much of the Landecker et al. (1989) argument for interaction is based on features that are not visible in the CGPS data. In Fig. 12 we present the velocity maps for H I emission in the region of VRO 42.05.01 and OA 184. The velocities go from $-20.5 \mathrm{~km} \mathrm{~s}^{-1}$ to $-43.5 \mathrm{~km} \mathrm{~s}^{-1}$. We plotted this range of velocities because Landecker et al. (1989) found that the systemic velocity of the SNR is $\sim-34 \mathrm{~km} \mathrm{~s}^{-1}$. Landecker et al. (1989) list a series of H I features, but some do not appear in the CGPS data:

- They locate a minimum of H I emission coincident with the position of VRO 42.05.01. at $-28 \mathrm{~km} \mathrm{~s}^{-1}$, which they identify with the interstellar cavity that the SNR has re-energised. In the CGPS data there is not a minimum at these velocities, rather the emission keeps decreasing at velocities more negative than $-28 \mathrm{~km} \mathrm{~s}^{-1}$.

- They find that the cavity that most closely resembles the shape of the SNR is at $-36 \mathrm{~km} \mathrm{~s}^{-1}$. Although we see a hole of emission in the CGPS data at those velocities in the position of the SNR, it is not evident that the shape of the cavity matches the shape of the SNR.

- They see a semi-circular arc defined by the continuum shell that appears to be completed by an $\mathrm{H}$ I feature at $-38 \mathrm{~km} \mathrm{~s}^{-1}$.

- They see a structure at $-42 \mathrm{~km} \mathrm{~s}^{-1}$ ("structure $\mathrm{K}$ " in their paper) that extends over the surface of the wing and that allegedly is accelerated gas associated with the cavity.

- They also find an expanding half shell from -34 to $-40 \mathrm{~km} \mathrm{~s}^{-1}$ ("structure J"), which they attribute to the rings formed as the shell is cut along the velocity axis.

The CGPS images are noise-limited with an rms brightness temperature $\Delta T_{B} \sim 3 \mathrm{~K}$ in each $0.82 \mathrm{~km} \mathrm{~s}^{-1}$ channel (Taylor et al. 2003), and so in principle should be able to detect emission from the features described in Landecker et al. (1989). In addition to these CGPS data not being clearly indicative of interaction, there do not appear to be two different neutral hydrogen density conditions that cut across the remnant at its alleged systemic velocity parallel to the Galactic plane.

The remnant, however, is at the boundary of a large polarised bubble visible in the CGPS polarisation data (Kothes \& Landecker 2004). The large structure, with an angular size of $9^{\circ}$, is clearly visible in the Stokes $U$ and $Q$ images, but only barely in polarised intensity and has no counterpart in Stokes $I$, which means it is likely a Faraday screen. The breakout boundary of the SNR (the line between the shell and the wing) coincides with the edge of the polarisation bubble. The authors 

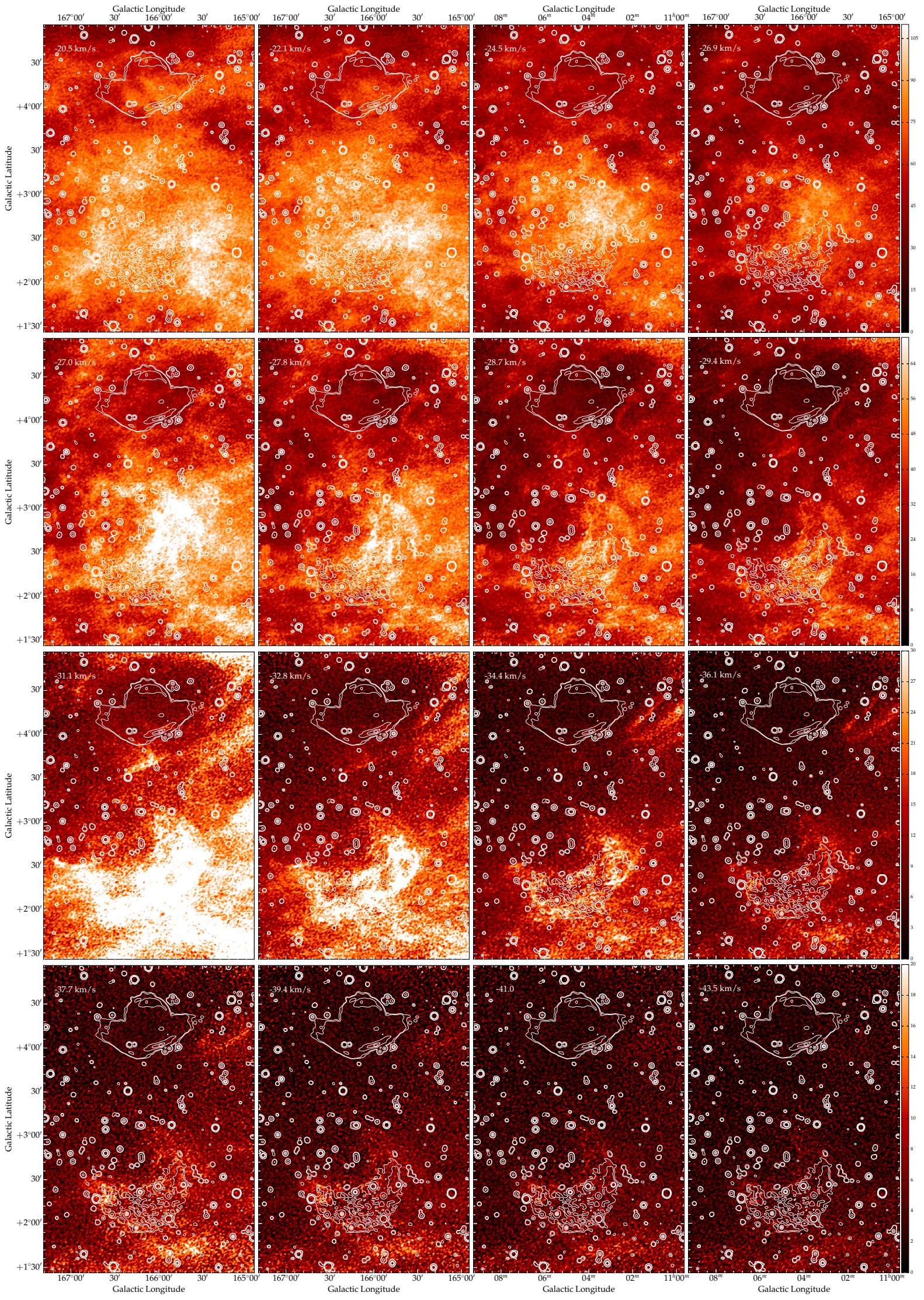

Fig. 12. Velocity distribution of HI emission towards VRO 42.05 .01 and OA 184. The data cube was taken from the CGPS. The radio contours at $143 \mathrm{MHz}$ from the LOFAR map in this work are overlaid in white. The wedges in the upper left corner of each plot correspond to the velocity of each slice. The brightness temperature $T_{\mathrm{B}}$ colour scale is the same for each row and goes as indicated by the colour bar to the left of each row, but it is not the same between rows. The ranges are as follows: first row: $0-110 \mathrm{~K}$; second row: $0-70 \mathrm{~K}$; third row: 0-30 K; and fourth row: 0-20 K. We have saturated the emission from OA 184 (bottom) to highlight the fainter emission in the surroundings of VRO 42.05.01. 
propose that it might be the remains of an old SNR whose highly compressed magnetic field still affects the background emission. This would be a way of having an environment with two sharply different densities.

In summary, many models that explain observed properties of VRO 42.05.01 are based on the SNR expanding into and interacting with media of different densities. However, most of the arguments in favour of association are based on morphological similarities and positional coincidence, and there is still no conclusive physical proof that the conditions around the remnant are in fact so inhomogeneous.

\section{Interpretation}

In the interpretation we discuss some possibilities for the spectral shape and spatial morphology of VRO 42.05.01.

\subsection{Possible reasons why VRO 42.05.01 has a curved spectrum at low frequencies}

In this section, we elaborate on some possible causes for the curved spectrum of VRO 42.05.01. In fact, many MM SNRs have radio spectral indices that are flatter than the canonical $\alpha=0.5$ predicted by standard shock acceleration theory (Bell 1987), and there is still no satisfying explanation for the existence of values of $\alpha<0.5$. Therefore, to explain the radio spectrum of VRO 42.05.01 we need a mechanism that is responsible for a flat spectrum with low-frequency steepening. We explore the following scenarios: high compression ratio shocks, two electron populations with different spectral indices that dominate at different frequencies, two shock regimes, and re-acceleration of previously accelerated electrons. We favour the high compression ratio shocks scenario, as it explains both how the flat spectrum originates and its steepening at low frequencies. However, we only briefly sketch it as a possible cause for the observed properties of VRO 42.05.01, and we caution that detailed simulations are required for it to be a convincing explanation of the presence of flat spectral indices in MM remnants.

\subsubsection{High compression ratio shocks}

The shock compression ratio $\chi$ is the ratio of postshock to preshock densities. For linear diffusive shock acceleration (DSA), the electron energy index $p$ is independent of energy and depends only on the shock compression ratio. For an electron distribution with $N(E) \mathrm{d} E=\kappa E^{-p} \mathrm{~d} E$, where the radio spectral index is $\alpha=\frac{p-1}{2}$, test-particle DSA predicts $p=\frac{\chi+2}{\chi-1}$ (Bell 1978). A strong shock has $\chi=4$, corresponding to $\alpha=0.5$.

Radiative shocks can give rise to high compression ratios, as the surrounding medium compresses the plasma while it loses energy. For isothermal shocks, compression ratios go as the square of the Mach number $\left(\chi=\gamma M^{2}\right.$, Draine 2011), so in principle very high compression ratios can be achieved even for modest Mach numbers, although in these cases the compressed magnetic field is likely to provide pressure support. Radiative shocks can be seen in the optical as bright, thin filaments. In many SNRs only parts of the shells are radiative, which may be the result of a non-uniform medium; the portions of the remnant expanding into a denser medium enter the radiative phase earlier and are thus visible in the optical (Blondin et al. 1998).

In these environments, the high $\chi$ does not happen immediately at the shock, but in an extended region behind it (Raymond 1979). Electrons of different energies are scattered further within the region of increasing $\chi$, and hence feel higher effective compression ratios. This is somewhat analogous to the mechanism of non-linear shock acceleration upstream of the shock, only this case affects the downstream region. The idea behind this mechanism as an explanation for the low-frequency steepening of the flat radio spectrum of VRO 42.05.01 is that as electrons of varying energies scatter back and forth along the shock front, they sample different compression ratios and therefore have a different value of the spectral index. The low-frequency electrons scatter closer to the shock front, experience a lower compression ratio, and so have a correspondingly higher index.

The diffusion length scale for an electron accelerated to very high energies is

$l_{\mathrm{diff}}=\frac{D}{v}=\frac{1}{3} \eta \frac{E c}{e B} \frac{\chi}{v_{\mathrm{sh}}}$

where $D$ is the diffusion coefficient, $v=\frac{v_{\text {sh }}}{\chi}$ is the velocity upstream of the shock, $\eta$ is a parametrisation factor $(\eta=1$ corresponds to Bohm diffusion), $E$ is the energy of the electrons, $c$ is the speed of light, $e$ is the elementary charge, and $B$ is the magnetic field. For synchrotron electrons, the critical emitting frequency is $v_{\mathrm{c}}=1.8 \times 10^{18} \mathrm{E}^{2} \mathrm{~B}$ (Ginzburg \& Syrovatskii 1965), and so we can expect that at $150 \mathrm{MHz}$ the electrons emitting synchrotron radiation in VRO 42.05.01 have a diffusion length-scale of

$l_{\text {diff }}=1.2 \times 10^{15} \eta\left(\frac{B}{10 \mu \mathrm{G}}\right)^{-1}\left(\frac{\chi}{4}\right)\left(\frac{v_{\mathrm{sh}}}{200 \mathrm{~km} \mathrm{~s}^{-1}}\right)^{-1} \mathrm{~cm}$,

and at $1500 \mathrm{MHz}$ they have instead

$l_{\text {diff }}=3.8 \times 10^{15} \eta\left(\frac{B}{10 \mu \mathrm{G}}\right)^{-1}\left(\frac{\chi}{4}\right)\left(\frac{v_{\mathrm{sh}}}{200 \mathrm{~km} \mathrm{~s}^{-1}}\right)^{-1} \mathrm{~cm}$.

In the models of Raymond (1979), for cooling shocks in the interstellar medium, the temperature conditions in the shock vary roughly an order or magnitude between the length scales of $1 \times 10^{15} \mathrm{~cm}$ and $4 \times 10^{15} \mathrm{~cm}$ (see e.g. Figs 1 and 2 in Raymond 1979). This could account for the difference in spectral index at $150 \mathrm{MHz}$ and $1500 \mathrm{MHz}$.

Of course, this is a simple order of magnitude estimate. The Raymond (1979) models assumed an ambient density of $n=10 \mathrm{~cm}^{-3}$, which seems rather high for VRO 42.05.01, and we do not know what is an appropriate value of $\eta(\eta \lesssim 10$ would correspond to a very turbulent medium). In practice, nonlinear effects can also be important (Ellison \& Eichler 1985). Onić (2013) linked high compression rations with flat spectra for radiative shocks. However, the situation of high compression ratios downstream of the shock has not been, to our knowledge, exhaustively dealt with in the theoretical literature, unlike the upstream case. We simply present this scenario as a possible explanation for the observed behaviour of the radio spectrum of VRO 42.05.01, and we note that further analysis is required both from theoretical and observational standpoints.

\subsubsection{Different electron populations and different shock regimes}

A natural explanation for variations in the radio spectral index is that there are two populations of emitting electrons, one with a steeper index and one with a flatter index, and each dominates at different frequency regimes. We find in Sect. 4 that the region with fainter radio emission has a roughly constant spectral index at low radio frequencies, and that the brighter regions steepen abruptly between $408 \mathrm{MHz}$ and $143 \mathrm{MHz}$. If this is due to two 

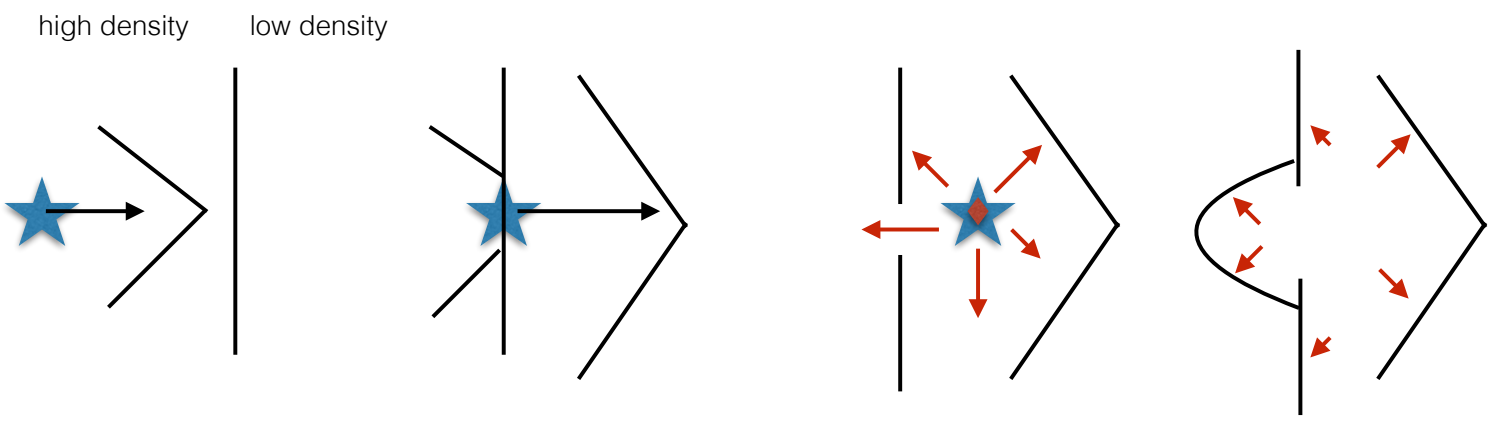

Fig. 13. Cartoon depicting our proposed scenario for the formation of VRO 42.05 .01 as discussed in Sect. 5.2. The progenitor of VRO 42.05.01 would have been travelling between media of two different densities, at a speed approximately 1.15 times the local sound speed in the ISM in the low-density region, creating a Mach cone of $120^{\circ}$. When the supernova explosion occurred, the ejecta would have expanded in the triangular cavity caused by the bow shock, and through the small cavity to the high-density region.

electron populations, the steep index population that becomes more dominant at low frequencies would need to be confined within the brightest regions.

The two electron populations can be due to different shock regimes that accelerate electrons with different energy indices and that are both present in the remnant. This would require that, in addition to the radiative shocks that outline the entire shell as evidenced from optical emission, there is another type of shock in the brightest regions. Another circumstance that can give rise to two different electron populations is that ISM electrons are compressed and accelerated (i.e. the van der Laan mechanism, van der Laan 1962). In this way, both the shock-accelerated and the compressed electrons are present, and each might have an intrinsically different spectral index. Rogers \& Bowman (2008) proposed that interstellar electrons have $\alpha=0.5 \pm 0.1$ between 100 and $200 \mathrm{MHz}$, which would mean that interstellar electrons contribute the steeper spectral component and SNR electrons contribute the flatter spectral component (Fig. 9 shows that $\alpha=0.37$ excluding the data point at $143 \mathrm{MHz}$ ).

The presence of two electron populations can account for the steepening of the spectrum, but we still lack an explanation as to how the population with a spectral index $\alpha<0.5$ arises.

\subsubsection{Re-acceleration}

Another method for creating a flat-spectrum electron population is the re-acceleration scenario proposed in Uchiyama et al. (2010). They showed that in MM SNRs interacting with molecular clouds, the re-acceleration of pre-existing cosmic-rays by DSA at a cloud shock is sufficient to power the observed $\gamma$-ray emission through the decay of neutral pions. These authors proposed that the radio emission may be enhanced by the presence of secondary electrons and positrons, that is, the products left over from the decay of charged pions. According to this model, the synchrotron radiation from the secondary electrons contributes most of the emission at lower radio frequencies, whereas that from the primary electrons (which naturally have a steeper spectrum) contributes most of the emission at higher radio frequencies. This model is at odds with our observations, since it predicts a flat, rather than a steep, spectrum at LOFAR frequencies.

\subsection{Is there a bow shock in VRO 42.05.01?}

The morphology of VRO 42.05.01 is extremely puzzling, and none of the proposed scenarios explain all of the observed properties of the source. In particular, the shape of the wing is vexing -it consists of almost straight lines forming an isosceles triangle with a vertex angle of $120^{\circ}$ with all sides emitting in the optical and radio, requiring sharp boundaries in all directions. The models in the Landecker et al. (1982) and Pineault et al. (1985, 1987) papers suggest that the explosion happened in the denser environment of the shell and eventually part of the shock broke out to a lower density region, forming the wing. This scenario cannot explain that the wider side of the wing emits strongly in optical and radio.

In this rather speculative section we present a modification of this scenario that could explain the sharp outline of the wing and the fact that it dissipates in the base angles.

Consider a massive star that is moving supersonically in a dense ISM region and that crosses the boundary to a more diffuse region perpendicularly, creating a small hole in the boundary separating the two regions. This star causes a bow shock. If the star is moving at 1.15 times the ISM sound speed, it forms a Mach cone of angle $120^{\circ}$.

If the star explodes shortly after crossing the boundary between the dense and diffuse regions, it will have exploded in a triangular cavity (the sides of the triangles are caused by the sides of the Mach cone, and the boundary between the two density regions), forming the wing. The base of the triangle will have a cavity due to the passage of the star, where the ejecta could expand into the higher density medium, more slowly than they do in the low-density triangle of the wing. This would explain the spherical, small shape of the shell. We depict this sequence of events in a cartoon in Fig. 13.

This scenario needs to be explored further with hydrodynamical simulations in order to be credible. Moreover, it shares the main caveat of the Landecker et al. (1982) and Pineault et al. $(1985,1987)$ model; namely that no conclusive evidence for a sharp density gradient has been found, as discussed in Sect. 4.3. Targeted, sensitive molecular line observations towards the edges of this SNR could convincingly establish interaction between the remnant and a neighbouring molecular cloud, or at least determine that there is in fact a molecular cloud in the environment of the remnant. Barring these limitations, we think that this is a plausible modification to the traditional model for the shape of VRO 42.05.01 that can explain more of the observed features without any additional difficulty.

\section{Conclusions}

In this paper we present a LOFAR map of the region of the Galactic plane centred at $l=166^{\circ}, b=3.5^{\circ}$ at $143 \mathrm{MHz}$, with a resolution of $148^{\prime \prime}$ and an rms noise of $4.4 \mathrm{mJy} \mathrm{bm}^{-1}$. Our map is sensitive to scales as large as $6^{\circ}$. We also make use of archival higher 
frequency radio images from the CGPS at 408 and $1420 \mathrm{MHz}$, IRAS infrared observations, and optical data. We analyse the two extended sources in our field, SNR VRO 42.05.01 and the H II region OA 184. We conclude the following:

1. VRO 42.05.01 has a higher flux density at LOFAR HBA frequencies than expected from reported values of the radio spectral index, resulting in a pretty significant increase of its radio spectral index at low frequencies, from the $\alpha=$ $0.36 \pm 0.06$ derived in Leahy \& Tian (2005) from observations at $408 \mathrm{MHz}$ and $1420 \mathrm{MHz}$, to $\alpha=0.49 \pm 0.02$ when we include the LOFAR flux density measurement at $143 \mathrm{MHz}$.

2. The flux density of OA 184 at LOFAR HBA frequencies agrees very well with the spectral index found by earlier works, further supporting the status of the source as an H II region.

3. The synchrotron tongue to the west of OA 184 does not have a more apparent radio luminosity at $143 \mathrm{MHz}$ than at $1420 \mathrm{MHz}$ and matches structures in the infrared and neutral hydrogen maps. We suggest that it is not a synchrotron source, but rather thermal in nature.

4. The faint diffuse emission in VRO 42.05.01 appears to have a constant spectral index of $\alpha=0.48 \pm 0.2$ between 143 and $2695 \mathrm{MHz}$. The two very bright regions north and west of the source have a remarkably flat spectral index between 408 and $2695 \mathrm{MHz}$ and steepen dramatically at LOFAR frequencies.

5. The radio and optical morphologies of VRO 42.05.01 are remarkably well matched, except for a region of excess optical emission in the interface of the shell and the wing. This region has overall a flatter spectral index than the rest of the SNR.

6. We explore several possible reasons for the spectral index of VRO 42.05.01 to steepen at low radio frequencies. We favour an explanation whereby the radiative shocks have high compression ratios, and electrons of different energies probe different length scales across the shocks, therefore sampling regions of different compression ratios.

7. We propose that VRO 42.05.01 is the remnant of a star that was moving supersonically in an inhomogeneous medium. The wing is explained as the expansion of the ejecta in the Mach cone formed by the progenitor star.

VRO 42.05.01 is a mysterious source, and, as is often the case, our new observations leave many questions about its nature unanswered. Molecular observations are required in order to unambiguously establish physical interaction between the remnant and its environment, and hydrodynamical simulations are key to understanding how the source developed its peculiar shape. Further observations of SNRs with the current generation of lowfrequency radio interferometers will shed light on how unique a feature the observed low-frequency steepening is to VRO 42.05.01 or to the mixed-morphology class. Exploring this regime is useful not only because it is where SNRs are the brightest, but also because both the environment and the physical processes at play in the shock can have an imprint on the low-frequency spectrum as departures from the synchrotron power law shape.

Acknowledgements. We thank the unnamed journal referee whose helpful and engaging comments improved the paper. The work of MA and JV is supported by a grant from the Netherlands Research School for Astronomy (NOVA). GJW gratefully acknowledges support from The Leverhulme Trust. This paper is based (in part) on data obtained with the International LOFAR Telescope (ILT) LOFAR (van Haarlem et al. 2013) is the LOw Frequency ARray designed and constructed by ASTRON. It has facilities in several countries, which are owned by various parties (each with their own funding sources) and are collectively operated by the ILT foundation under a joint scientific policy. LOFAR data reduction used the DRAGNET GPU cluster (at the CIT in Groningen), which was funded by the European Research Council under the European Union's Seventh
Framework Programme (FP7/2007-2013) / ERC grant agreement nr. 337062 (PI: Hessels). The research presented in this paper has used data from the Canadian Galactic Plane Survey, a Canadian project with international partners, supported by the Natural Sciences and Engineering Research Council.

\section{References}

Ackermann, M., Ajello, M., Allafort, A., et al. 2013, Science, 339, 807 Araya, M. 2013, MNRAS, 434, 2202

Bell, A. R. 1978, MNRAS, 182, 147

Bell, A. R. 1987, MNRAS, 225, 615

Biggs, J. D., \& Lyne, A. G. 1996, MNRAS, 282, 691

Blondin, J. M., Wright, E. B., Borkowski, K. J., \& Reynolds, S. P. 1998, ApJ, 500,342

Bocchino, F., Miceli, M., \& Troja, E. 2009, A\&A, 498, 139

Briggs, D. S. 1995, AAS Meeting Abstracts, 112.02

Burrows, D. N., \& Guo, Z. 1994, ApJ, 421, L19

Chen, Y., \& Slane, P. O. 2001, ApJ, 563, 202

Draine, B. T. 2011, Physics of the Interstellar and Intergalactic Medium (Princeton University Press)

Ellison, D. C., \& Eichler, D. 1985, Phys. Rev. Lett., 55, 2735

Fesen, R. A., Blair, W. P., \& Kirshner, R. P. 1985, ApJ, 292, 29

Foster, T., Kothes, R., Sun, X. H., Reich, W., \& Han, J. L. 2006, A\&A, 454, 517

Fürst, E., Reich, W., Reich, P., \& Reif, K. 1990, A\&AS, 85, 691

Gaensler, B. M., Chatterjee, S., Slane, P. O., et al. 2006, ApJ, 648, 1037

Gao, X. Y., Han, J. L., Reich, W., et al. 2011, A\&A, 529, A159

Ginzburg, V., \& Syrovatskii, S. 1965, ARA\&A, 3, 297

Giuliani, A., et al. 2011, ApJ, 742, L30

Green, D. A. 2017, VizieR Online Data Catalog: VII/278

Guo, Z., \& Burrows, D. N. 1997, ApJ, 480, L51

Huang, Y.-L., \& Thaddeus, P. 1986, ApJ, 309, 804

Hwang, U., Petre, R., \& Hughes, J. P. 2000, ApJ, 532, 970

Intema, H. T., Jagannathan, P., Mooley, K. P., \& Frail, D. A. 2017, A\&A, 598, A78

Kothes, R., \& Landecker, T. L. 2004, in The Magnetized Interstellar Medium, eds. B. Uyaniker, W. Reich, \& R. Wielebinski, 33

Kothes, R., Fedotov, K., Foster, T. J., \& Uyanıker, B. 2006, A\&A, 457, 1081

Landecker, T. L., Pineault, S., Routledge, D., \& Vaneldik, J. F. 1982, ApJ, 261, L41

Landecker, T. L., Pineault, S., Routledge, D., \& Vaneldik, J. F. 1989, MNRAS, 237,277

Lazendic, J. S., \& Slane, P. O. 2006, ApJ, 647, 350

Leahy, D., \& Tian, W. 2005, A\&A, 440, 929

Lorimer, D. R., Lyne, A. G., \& Camilo, F. 1998, A\&A, 331, 1002

Mahony, E. K., Morganti, R., Prandoni, I., et al. 2016, MNRAS, 463, 2997

Matsumura, H., Uchida, H., Tanaka, T., et al. 2017, PASJ, 69, 30

Noordam, J. E. 2004, in Ground-based Telescopes, ed. J. M. Oschmann, Jr., Proc. SPIE, 5489, 817

Offringa, A. R., McKinley, B., Hurley-Walker, N., et al. 2014, MNRAS, 444, 606 Onić, D. 2013, Ap\&SS, 346, 3

Pineault, S., Pritchet, C. J., Landecker, T. L., Routledge, D., \& Vaneldik, J. F. 1985, A\&A, 151, 52

Pineault, S., Landecker, T. L., \& Routledge, D. 1987, ApJ, 315, 580

Raymond, J. C. 1979, ApJS, 39, 1

Rho, J., \& Petre, R. 1998, ApJ, 503, L167

Rogers, A. E. E., \& Bowman, J. D. 2008, AJ, 136, 641

Sezer, A., \& Gök, F. 2012, MNRAS, 421, 3538

Shimwell, T. W., Röttgering, H. J. A., Best, P. N., et al. 2017, A\&A, 598, A104 Shimwell, T. W., Tasse, C., Hardcastle, M. J., et al. 2018, A\&A, 622, A1, (LOFAR SI)

Taylor, A. R., Gibson, S. J., Peracaula, M., et al. 2003, AJ, 125, 3145 Troja, E., Bocchino, F., Miceli, M., \& Reale, F. 2008, A\&A, 485, 777 Uchiyama, Y., Blandford, R. D., Funk, S., Tajima, H., \& Tanaka, T. 2010, ApJ, 723, L122

van den Bergh, S., Marscher, A. P., \& Terzian, Y. 1973, ApJS, 26, 19 van der Laan, H. 1962, MNRAS, 124, 125

van Haarlem, M. P., Wise, M. W., Gunst, A. W., et al. 2013, A\&A, 556, A2 van Weeren, R. J., Williams, W. L., Hardcastle, M. J., et al. 2016, ApJS, 223, 2 Vessey, S. J., \& Green, D. A. 1998, MNRAS,294, 607

Vink, J. 2012, A\&ARv, 20, 49

Williams, W. L., van Weeren, R. J., Röttgering, H. J. A., et al. 2016, MNRAS, 460, 2385

Wright, E. L., Eisenhardt, P. R. M., Mainzer, A. K., et al. 2010, AJ, 140, 1868 Yamaguchi, H., Tanaka, M., Maeda, K., et al. 2012, ApJ, 749, 137

Yatawatta, S., Zaroubi, S., de Bruyn, G., Koopmans, L., \& Noordam, J. 2008, ArXiv e-prints [arXiv:0810.5751]

Zhou, P., \& Vink, J. 2018, A\&A, 615, A150 


\section{Appendix A: Narrow-band images}

We present as an Appendix the three narrow-band LOFAR HBA images.
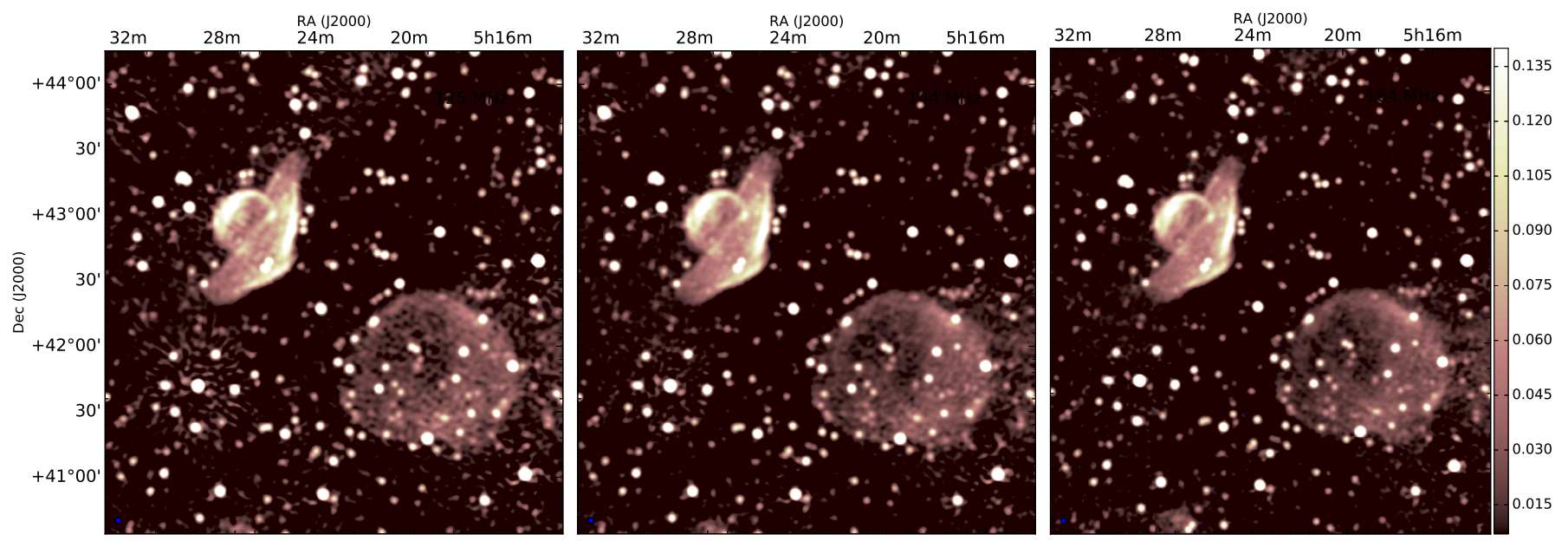

Fig. A.1. Uv-matched, narrow-band images at $125 \mathrm{MHz}, 144 \mathrm{MHz}$, and $164 \mathrm{MHz}$. All images are on the same colour scale. 